\title{
FERMILAB-CONF-05-151-AD DETERIORATION OF THE SKEW QUADRUPOLE MOMENT IN TEVATRON DIPOLES OVER TIME*
}

\author{
M. J. Syphers and D. J. Harding, Fermilab, Batavia, IL 60150, USA
}

\begin{abstract}
During the 20 years since it was first commissioned, the Fermilab Tevatron has developed strong coupling between the two transverse degrees of freedom. A circuit of skew quadrupole magnets is used to correct for coupling and, though capable, its required strength has increased since 1983 by more than an order of magnitude. In more recent years changes to the Tevatron for colliding beams operation have altered the skew quadrupole corrector distribution and strong local coupling become evident, often encumbering routine operation during the present physics run. Detailed magnet measurements were performed on each individual magnet during construction, and in early 2003 it was realized that measurements could be performed on the magnets in situ which could determine coil movements within the iron yoke since the early 1980's. It was discovered that the superconducting coils had become vertically displaced relative to their yokes since their construction. The ensuing systematic skew quadrupole field introduced by this displacement accounts for the required corrector settings and observed beam behavior. An historical account of the events leading to this discovery and progress toward its remedy are presented.
\end{abstract}

\section{INTRODUCTION}

During the start-up of Run II of the Tevatron Collider program, several issues surfaced which were not present, or not seen as detrimental, during Run I. These included the repeated deterioration of the closed orbit requiring orbit smoothing every two weeks or so, the inability to correct the closed orbit to desired positions due to various correctors running at maximum limits, regions of systematically strong vertical dipole corrections, and the identification of very strong coupling between the two transverse degreesof-freedom. It became apparent that many of the problems being experienced operationally were connected to a deterioration of the main dipole magnet alignment, and remedial actions were undertaken.[1] However, the alignment alone was not enough to explain the corrector strengths required to handle transverse coupling.

Strong coupling had been an issue in the Tevatron during Run I. The start-up of Run Ib was complicated by what was later discovered to be a rolled triplet quadrupole magnet in one of the Interaction Regions.[2]. This led to a reduction in luminosity of nearly $50 \%$, as well as operational confusion until it was uncovered. With this history, several studies were performed early in Run II to search for

\footnotetext{
* Work supported by the U.S. Department of Energy under contract No. DA-AC02-76CH03000.
}

similarly strong local coupling sources, but without success. The Tevatron is equipped with one large coupling correction circuit, and a few individual skew quadrupole correctors. The strong corrector settings were indicative of a much larger problem than a single rolled magnet, but yet the locality of the error was hard to deduce from the setting of a global correction system.

\section{Strong Systematic Steering Correction}

In late 2002 regions of the Tevatron were found to contain vertical steering magnets whose average strength was required to be non-zero in order to produce a smooth trajectory as seen on the Beam Position Monitors. Compared to the $0.7 \mu \mathrm{rad}$ average horizontal steering corrector strength, the vertical correctors had a ring-wide average of about $16 \mu \mathrm{rad}$, and areas of the Tevatron had strengths of 70$90 \mu \mathrm{rad}$ averaged over distances of $400 \mathrm{~m}$ or so. At $1 \mathrm{TeV}$, the maximum strength of a corrector is a little more than $100 \mu \mathrm{rad}$ and so the available correction for general beam steering was limited in these locations. The interpretation of this effect was that these areas contained magnets which were systematically rolled toward the inside of the tunnel. This was verified by magnet roll angle measurements performed in October 2002, and January 2003.

The systematic corrections produce a "scalloped" vertical trajectory through the bending regions.[3] Though the distributed beam position monitors might read zero displacements, the beam will actually undergo $\sim 0.5 \mathrm{~mm}$ excursions through these regions, assuming the magnets are rolled about the beampipe axis. The fact that they are actually rolled about a different axis closer to the floor means that the beam trajectory may be closer to $1 \mathrm{~mm}$ or more from the center of the magnet coil.

Since the Tevatron dipoles have a sextupole component, a systematic vertical offset will feed-down into a coupling term between the horizontal and vertical motion. The sextupole component is also known to vary as the logarithm of time due to persistent current effects at low field, hence the coupling would vary with time accordingly. It is thought that this effect explains much of the observed tune drift behavior during the Tevatron injection process, though it is not nearly enough to explain the large skew quadrupole corrector settings.

\section{Injection Experiment}

The transverse coupling generated by orbit feed-down through rolled dipole magnets and the estimated coupling due to observed quadrupole magnet rolls could not account for the strong correction required of the main skew quad circuit early in Run II. Taken together, these effects 


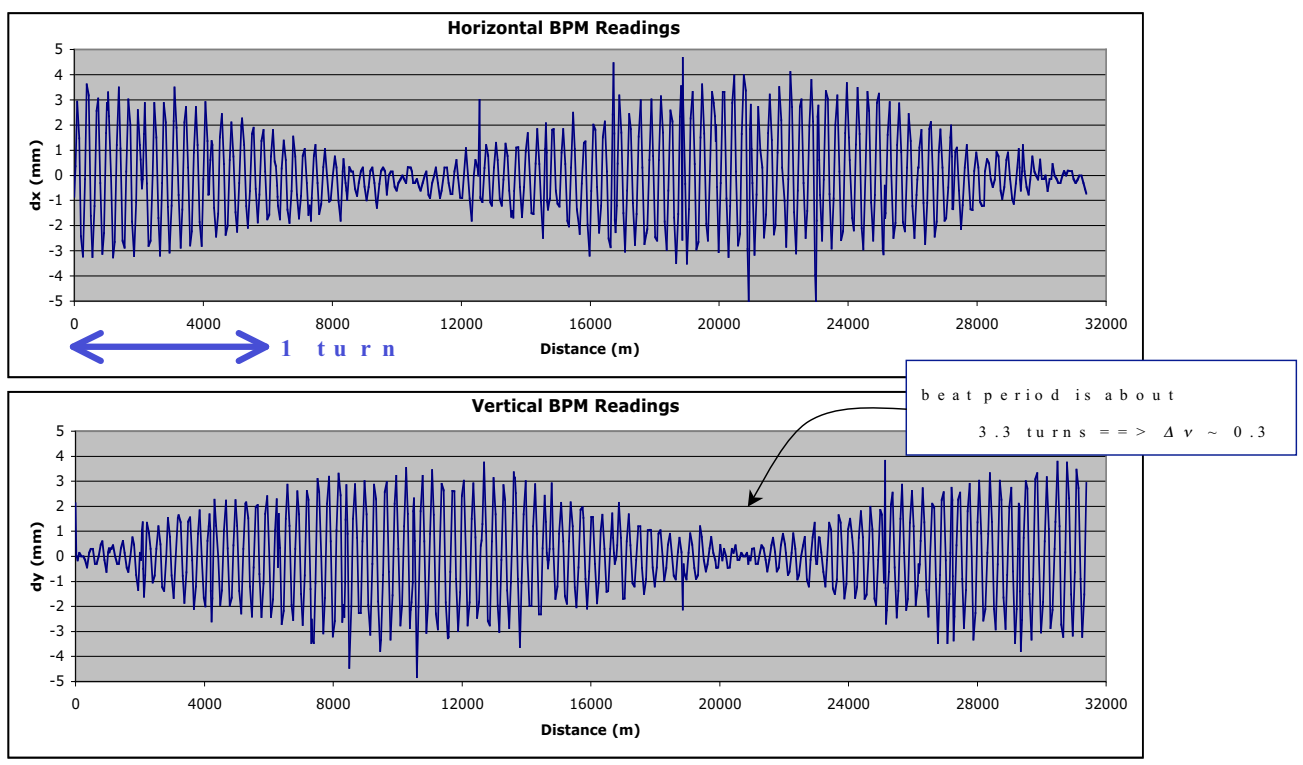

Figure 1: Data taken in February, 2003, showing horizontal motion coupling fully into the vertical plane in less than 2 turns. One revolution about the Tevatron is about $6283 \mathrm{~m}$; the data are for 5 consecutive turns.

were strong enough to explain a correction of the minimum difference between the two transverse tunes of amount $\Delta \nu \approx 0.03$. However, the setting required of the main skew quadrupole correction circuit to decouple the Tevatron was indicative of a minimum tune difference an order of magnitude larger. An experiment was performed[4] in which protons were injected into the Tevatron with all skew quadrupole correctors turned off. The result, given in Figure 1, shows pure horizontal motion being coupled completely into the vertical plane within about 1.5 revolutions.

The fact that the coupling builds up gradually and not at a a few localized sources is indicative of a uniformly distributed source of skew quadrupole fields. The 3-turn period of the coupling is consistent with a tune split of order 0.3 . It was quickly noted that a systematic skew quadrupole term, $a_{1} \equiv \partial\left(B_{x} / \partial x\right) / B_{0}$, would account for this behavior, and would need to be of order $a_{1}=1.5 \times 10^{-4} \mathrm{inch}^{-1}$, or 1.5 "units." [4]

\section{SEARCH FOR COUPLING SOURCES}

In January, 2003, as plans were being made to correct the rolled Tevatron dipoles, the question arose of ensuring that the cryostat and coils moved with the warm iron magnet yoke. It was noted that provision had been made during construction of the magnets for monitoring the cryostat movement through a measurement known as the "lift" using a depth gauge. To assess the viability of making these measurements in the tunnel and the stability of the cryostat over time, technicians measured 18 magnets during a day in February when the accelerator was down for maintenance. The original (paper) production records of the magnets were retrieved from off-site storage and the lift mea- surements extracted.

By the end of February the data had been analyzed and it was apparent that in those 18 magnets the coil had systematically dropped approximately $0.11 \mathrm{~mm}$ relative to the yoke. (See Figure 2.) After a bit of thought it was realized that this was probably due to "creep," slow inelastic deformation under pressure, in the G11/G10 blocks that support the coil within the cryostat. This change is enough to produce a skew quadrupole component in the field of about 1.15 "units." Indeed, the position had been adjusted during production to minimize the skew quadrupole.

Knowing that betatron coupling was a troubling issue in the Tevatron, but not knowing of the active calculations and measurements, one of the authors (Harding) sent e-mail to the other (Syphers): "If every Tevatron dipole had de-

\section{Sums and differences of cold lift changes}

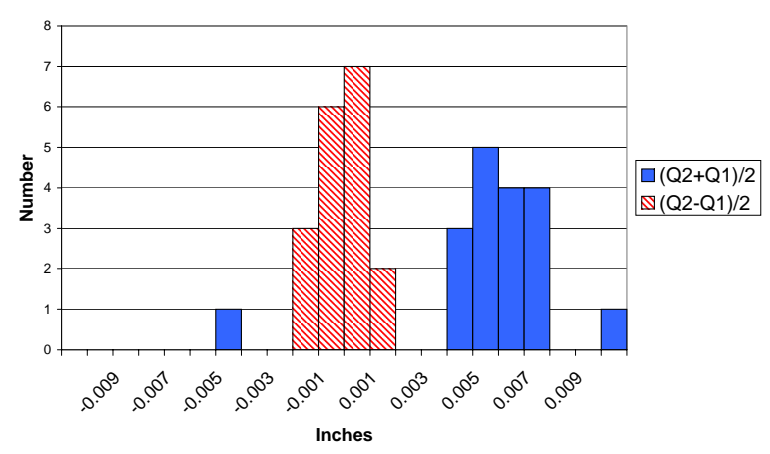

Figure 2: First Cold Lift Measurements. Q1 and Q2 are left and right measurements; a net sum indicates a vertical displacement; a net difference would indicate a rotated coil. 
veloped with age a skew quadrupole component, how big would it need to be to explain the effects that are currently seen?" When the authors conferred and discovered that they were taking about numbers that matched quite well, they and the others involved agreed to report the results and propose further studies.

The original calculations from the production era were repeated, affirming the linear relationship between displacement and skew quadrupole error term, $a_{1}=k \cdot y$, where $k$ is 10.6 units $/ \mathrm{mm}$. The magnets had been designed to allow adjustment of the coil position while the magnet was cold by adding and removing shims from the cryostat suspension system. During production this feature was used as a standard procedure to compensate for fabrication tolerances and mechanical differences between the top and bottom coil. Each of the almost 1000 dipoles (including spares) built for the Tevatron was measured cold and, if necessary, adjusted to remove the skew (and normal) quadrupole components and remeasured.

\section{FURTHER CONFIRMATIONS}

Once the Tevatron dipole magnets were considered to be the main source of the coupling, further verifications were performed using beam measurements as well as magnet measurements. It was determined that during Run I skew quadrupoles were removed from the main corrector circuit in the vicinities of the two major detectors. Thus, while the distribution of $a_{1}$ was essentially uniform around the ring, its correction was no longer. This led to a residual vertical dispersion wave which was measured and correctly interpreted, verifying our understanding.[4]

The lifts of an additional sample of 66 magnets were measured in the tunnel during a subsequent short maintenance period, confirming the original distribution. Similar changes in the coil position were measured in spare dipoles that had not been in service. Several spare dipoles were magnetically measured [5], confirming that the long term change in lift was reflected in the expected change in skew quadrupole.

With the recognition that reshimming the dipoles to remove the skew quadrupole was possible, more magnet studies were conducted and detailed plans were laid.[6] The position of the magnet cryostat and the coil inside is determined by two bolts through the bottom of the yoke 45 degrees from vertical that form a cradle at 9 stations down the length of the 6-meter magnet. These align with small G-11 blocks inside the cryostat that directly support the collared coil assembly, the blocks that we believe have deformed. Brass shims between the bolt and the cryostat allow reproducible adjustments of the position. Spring loaded cartridges ("smart bolts"), penetrating from the top, hold the cryostat in position against the lower bolts as the system contracts during cool down.

During production the results of shimming could be verified immediately. Measuring the lifts of each magnet, comparing them with the production values, and reshimming accordingly would have been fraught with possibilities for error. Examination of the production data quickly led us to the conclusion that it was not sufficiently reliable on a magnet-by-magnet basis to warrant the effort of individual treatment. Instead, it was decided to treat all magnets as though they had changed by the same amount. A standard shim change was agreed upon.

\section{REMEDIATIONS AND PRESENT STATUS}

With 774 dipole magnets in the Tevatron, and with 18 shim locations per magnet, it was clear that to fix the problem completely would take significant tunnel access time. Any piece-wise repair of the problem had to be performed with the skew quadrupole corrector system always in mind. Thus, patterns of magnet repairs were chosen which were consistent with the corrector system layout and which could be carried out in tolerable amounts of time.

The sensitivity of the operation led us to augment our usual quality assurance procedures with more formal analyses.[7] The sheer volume of measurements, $18 \mathrm{lift}$ measurements for each magnet before and after the reshimming, required extensive above ground monitoring in addition to the teams of technicians in the tunnel. Although the job in the tunnel is tedious, requiring the technicians to crawl over the magnets in tight quarters, a crew of three can comfortably reshim two magnets per day .

In the fall of 2003, during the annual accelerator shutdown, 106 dipoles were reshimmed in the neighborhood of the intersection regions. Another 12 magnets were reshimmed in March 2004 during a one-week shutdown. With the worth of the project demonstrated through improved accelerator performance, the fall 2004 shutdown saw a concentrated effort to reshim 412 more magnets. We plan to reshim the remaining 244 magnets during the next major shutdown, currently planned for the late fall of 2005 .

\section{REFERENCES}

[1] J.T. Volk, et al., "Tevatron Alignment Issues 2003-2004," these proceedings.

[2] J. Holt, et al., "Calculating Luminosity for a Coupled Tevatron Lattice," PAC95 (1996); N. Gelfand, "Coupling in the Tevatron," PAC95 (1996).

[3] M.J. Syphers, "Strong Systematic Steering Correction in Regions of the Tevatron," Fermilab Internal Report Beams-doc491 (2003).

[4] M.J. Syphers, et al., "Observations of Strong Transverse Coupling in the Tevatron," these proceedings.

[5] G. Ambrosio et al., "Results of an Investigation of the Skew Quadrupole Issue in Tevatron Dipoles," Fermilab Internal Report TD Note 03-045 (2003).

[6] D.J. Harding, et al., "Restoring the Skew Quadrupole Moment in Tevatron Dipoles," these proceedings.

[7] J.N. Blowers, et al., "Reshimming of Tevatron Dipoles; A Process-Quality and Lessons-Learned Perspective," these proceedings. 